\title{
REGULATION OF BLOOD PRESSURE LEVELS BY HEXAMETHONIUM BROMIDE AND MECHANICAL DEVICES
}

\author{
BY \\ P. A. RESTALL AND F. H. SMIRK \\ From the Department of Medicine, University of Otago, Dunedin, New Zealand \\ Received June 16, 1951
}

Probably the first studies of the pharmacology of quaternary ammonium compounds were made by Crum-Brown and Fraser in 1868. Burn and Dale (1915) found that, unlike nicotine, tetraethyl-ammonium chloride blocked autonomic ganglia without noticeable initial stimulation. Barlow and Ing (1948a and $b$ ), Paton and Zaimis (1948 a and b), and Balaban et al. (1949) studied the properties of some chemical relatives of tetra-ethyl-ammonium in which two quaternary nitrogen groups were separated by a chain of $\mathrm{CH}_{2}$ groups. It was found that decamethonium with ten $\mathrm{CH}_{2}$ groups was curare-form, and pentamethonium and hexamethonium with respectively five and six $\mathrm{CH}_{2}$ groups were predominantly ganglion blocking agents. For example, Paton and Zaimis (1949) showed that intravenous injection of pentamethonium iodide relaxed the cat's nictitating membrane which had been caused to contract by preganglionic stimulation. Pentamethonium iodide also prevented the pulse-slowing action of vagal stimulation. Kay and Smith (1950) showed that hexamethonium iodide lessened the secretory response to insulin-induced hypoglycæmia but did not prevent the response of gastric secretion to histamine. All of these results are consistent with the view that penta- and hexamethonium salts act chiefly by blocking autonomic ganglia.

Organe et al. (1949), and Arnold and Rosenheim (1949) showed that intravenous or intramuscular injections of pentamethonium produced falls of blood pressure in subjects with normal or raised blood pressure. The effect was similar to that of tetra-ethyl-ammonium chloride but lasted longer and was accompanied by fewer subjective sensations. These authors also noted postural hypotension, and Arnold and Rosenheim concluded that postural hypotension was likely to limit the value of the substance in treatment and that the most promising application was to peripheral vascular disease. Arnold et al. (1949) showed an increase in the flow through the fingers using a finger plethysmograph. Burt and Graham (1950) confirmed the existence of peripheral dilatation in the legs by skin temperature methods but they found little change in the circulation through the arms.

Restall and Smirk (1950) made therapeutic use of the postural hypotension to enhance the blood pressure lowering effect of penta- and hexamethonium in patients with high blood pressure. They reported encouraging results which further observations have confirmed (Smirk, 1950a and $b$; Smirk and Alstad, 1951). They report that a salt-free diet may enhance the effect of methonium salts, an effect of practical importance. Additional clinical reports have been published by Turner (1950), Saville (1950) and Campbell and Robertson (1950) with varying degrees of benefit.

The present paper is concerned with the circulatory effect of hexamethonium bromide (H.M.B.) in man. Many similar experiments have been made with pentamethonium bromide (P.M.B.) and no substantial difference in their effects has been detected.

\section{METHODS}

Plethysmographs used were modifications of those described by Drury and Jones (1927). Water at $33^{\circ} \mathrm{C}$. was employed as the medium for transmitting volume changes. In the case of the upper limb, the forearm was horizontal and about $20 \mathrm{~cm}$. below the angle of Louis. 
Venous pressures were measured in an antecubital vein by the method of Cohen (1936), the angle of Louis being taken arbitrarily as a zero point.

Ballistocardiograms were used to register induced changes in the cardiac output in the sitting posture. The apparatus will be described elsewhere.

The application of negative or positive pressure to the lower two-thirds of the body requires a suitable pump and a rigid air-tight container in which the patient stands or lies. The junction of the patient's chest to this container is achieved with sheet-plastic applied in such a way that the seal with the skin is actually made by the air pressure-negative or positive as the case may be (Fig. 1). It is essential to support this junction sheet, on the inside for negative pressures and on the outside for positive ones, as even with moderate pressures the total force exerted over its whole area is not inconsiderable. The equipment used to achieve this was made and assembled in the following way.
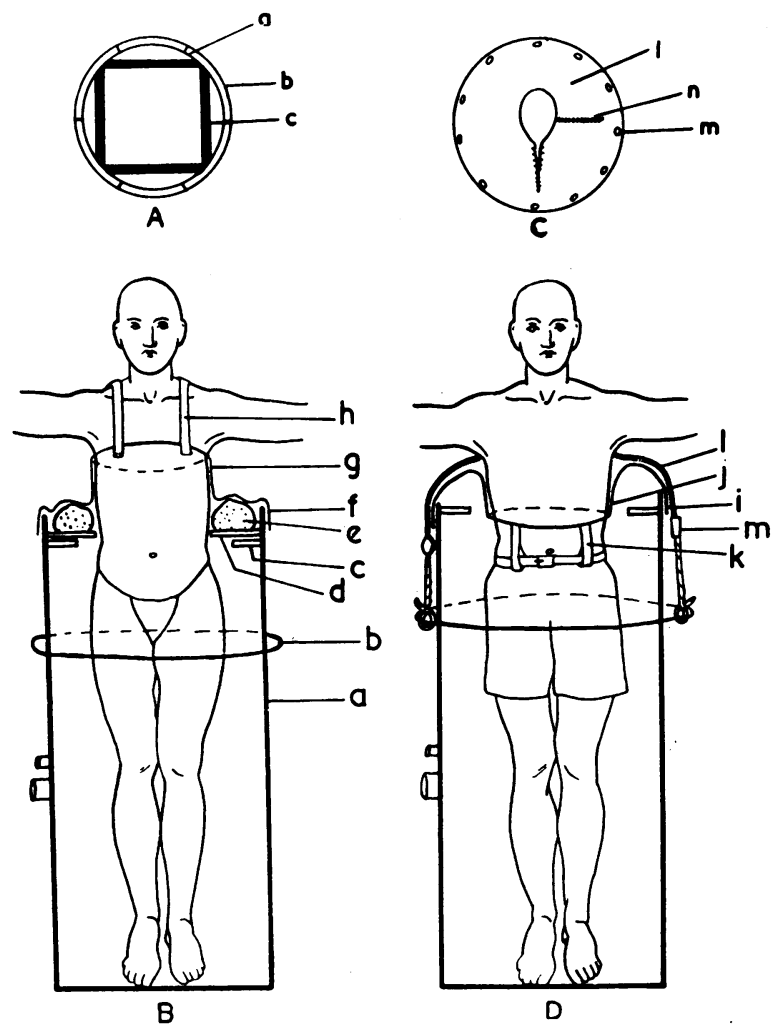

FIG. 1.-Devices for exercising negative $(A, B)$ and positive (C, D) air pressures upon the external surface of the lower two-thirds of the body.

Two tubes are let into the metal drum (a). The smaller is connected to a manometer and the larger $\left(\frac{3}{4}\right.$ inch) by wide-bore hose to a large capacity pump (Burdick, U.S.A.) variable for compression or suction at will.

For the application of a negative pressure (Fig. 1A and B) the subject, with the chest bare, stands in the metal drum (a). The axillae should clear the top by a few inches. A pair of plywood half circles (d) are now slipped into place resting inside the drum on the metal strips (c). They are cut so as to fill the gap between the metal drum and the patient's chest. A "sausage" (e) made of cloth filled with chaff is now placed on the plywood encircling the subject and is moulded so as to further fill the gap between the drum edge and the chest wall. Next the plastic tube $(f, g)$ is pulled 
down over the patient's head, the lower end (f) being sealed to the drum with sticking plaster or an elastic bandage. The upper end ( $\mathrm{g}$ ) will be but loosely applied to the chest and held up by the shoulder straps (h). This plastic tube with its shoulder straps is like a singlet but much wider at the bottom. If suction is now commenced (negative pressure should be used only with great caution if the patient is still erect) sealing occurs automatically if the plastic is momentarily held against the chest wall. The strain is taken by the shoulder straps and "sausage" on the plywood. There must be no large gaps between the patient and the drum or the plastic will balloon through and burst. With this method negative pressure of from $2-100 \mathrm{~mm}$. $\mathrm{Hg}$ are readily obtained. A good sealing usually occurs even at low pressures but rarely a smear of vaseline is necessary.

For the application of a positive pressure (Fig. 1C and D) a plastic tube (i,j) similar to that used for negative pressure experiments makes the seal between the patient's skin and the top of the drum. It is lightly held to the skin by a rubber bandage (not shown) and firmly bound to the outside of the drum. The plastic tube is prevented from ballooning under a positive pressure first by calico strips (k) holding down the free edge to a belt worn for the purpose, and secondly by a strong calico sheet (1) with a central hole to admit the patient's thorax. The size of the hole is adjusted by zip fasteners (n) so as to bring the sheet in close apposition to the chest wall. The perimeter of the sheet (1) is tied firmly by cords through the eyelet holes (m) to the iron hoop (b) on the drum. Pressures of $100 \mathrm{~mm}$. $\mathrm{Hg}$ are obtainable.

Further practical details are obtainable from the authors.

\section{RESULTS}

\section{Effect of Hexamethonium Bromide on Limb Volume}

Patients were at rest in the sitting posture with a forearm and a leg, to upper calf level, each in a plethysmograph. The forearm was horizontal and held some $20-30 \mathrm{~cm}$. below the angle of Louis throughout the experiment. When the leg and the arm volumes were approximately steady H.M.B. was injected in doses of $20 \mathrm{mg}$. intravenously or $20-50 \mathrm{mg}$. subcutaneously. Following injection, the leg volume increased. The arm volume sometimes increased and sometimes decreased (Fig. 2). The increase in leg volume in 8 observations varied but lay between 15 and $80 \mathrm{ml}$. per $2000 \mathrm{ml}$. of limb immersed in the water of the plethysmograph. The changes in arm volume in 8 observations ranged from an increase of $20 \mathrm{ml}$. to a decrease of $14 \mathrm{ml}$. Variation in the direction of change in forearm volume is not surprising. The comparatively small volume increase in the legs might be explained by the fact that an external counter-pressure is imposed on the leg by the water in the leg plethysmograph.

\section{Effect of H.M.B. on the Venous Pressure in High Blood Pressure, Congestive Heart Failure, and Superior Vena Cava Obstruction}

In hypertensives without heart failure following intravenous or, in a few instances, subcutaneous administration of $H . M . B$. there was a fall of $1-4 \mathrm{~cm}$. of water pressure and in some instances it was noted that the venous pressure began to fall before the arterial pressure had decreased. Observations were made also in patients with congestive heart failure whose venous pressures could be measured readily by inspection of the external jugular veins (Lewis, 1943). The venous pressure fell distinctly in all these patients and in 4 of them there was at the same time a decrease in breathlessness.

\section{Effect of an Externally Applied Counter-Pressure upon Postural Hypotension induced by H.M.B.}

If the increase in leg volume and the decrease in venous pressure induced by H.M.B. are to be explained in part by inability of vessel walls to resist the hydrostatic pressure of blood within them, any fall of blood pressure so induced should not be so great if rubber bandages are applied to the legs and abdomen. We found, however, that pressure on the abdomen or elastic binding of the legs including the thighs, or both procedures combined were not fully effective methods of restraining the 


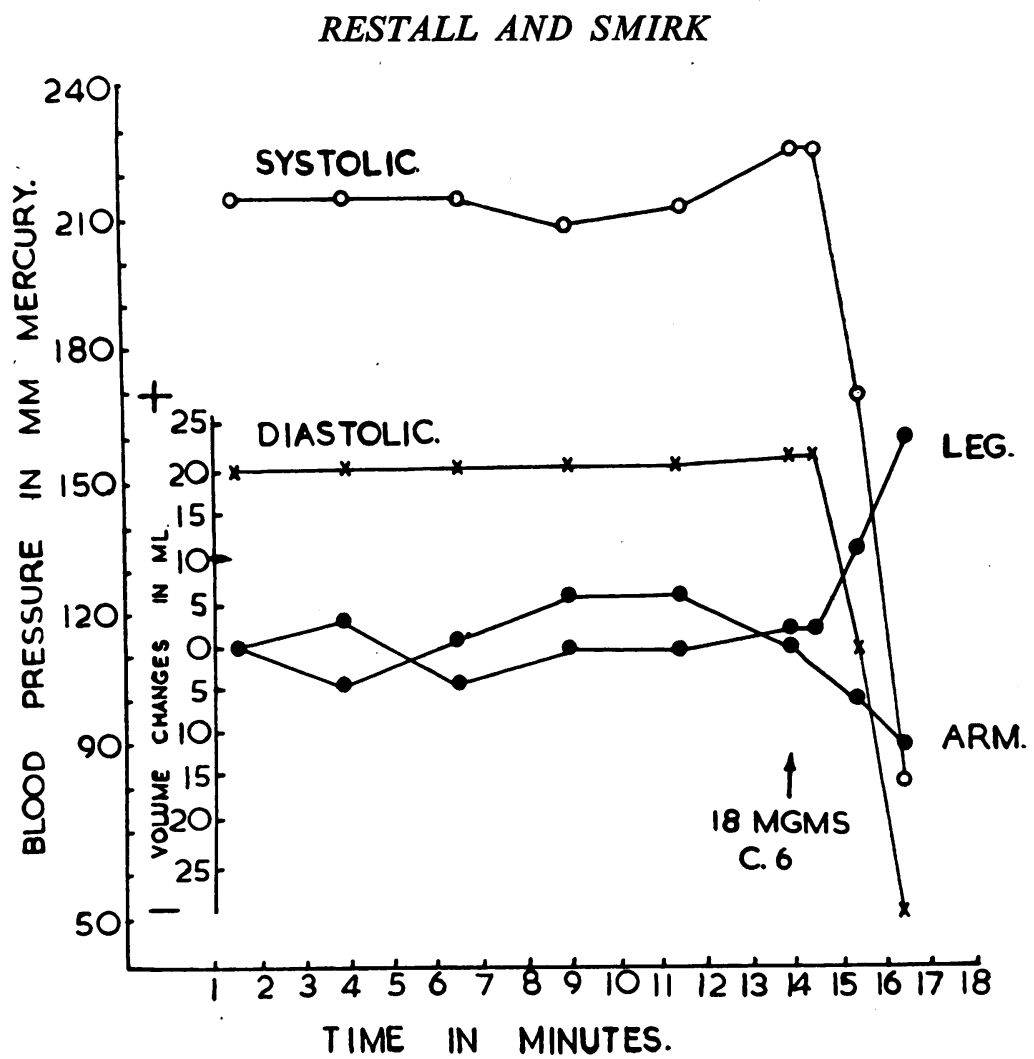

Fig. 2.-Effect of H.M.B. on limb volume.

postural hypotension after injections of H.M.B., though the extent of the blood pressure fall might be decreased by $30 / 10 \mathrm{~mm}$. $\mathrm{Hg}$.

This may be partly explained because at rest the pressure at any point in a vein or artery is equal to the general venous or arterial pressure plus the pressure of the column of blood extending vertically downwards from the angle of Louis to the point in the vessel under consideration. In the erect posture at rest it follows that the pressure in the veins at thigh level is less than the venous pressures near the ankle. Hence, if rubber bandages are applied with equal firmness to all parts of the legs, a degree of firmness that prevents over-filling of veins in the region of the ankle may obstruct the veins higher up.

That application of a uniform external pressure may actually prevent or delay the emptying of veins is readily seen in a simple mechanical model. A length of sausage skin, closed at one end, is tied on to a glass tube open at both ends. The sausage skin and the tube are filled with water and the skin suspended in the pressure bottle, the tube passing through a rubber stopper to the outside of the bottle. If the length of the sausage skin is $10 \mathrm{~cm}$., the pressure of water within is a gradient between 0 at the top and $10 \mathrm{~cm} . \mathrm{H}_{2} \mathrm{O}$ at the bottom. If now air is introduced from a reservoir, so that the pressure in the bottle is raised, expulsion of the water in the sausage skin does not occur, even though the air pressure may be many times greater than the pressure of water at the bottom of the sausage skin. What happens is that the upper part of the skin is compressed whilst the lower part remains distended with fluid, which either does not empty or does so extremely slowly. If water instead of air is introduced into the bottle we provide an external counterpressure which is graduated, being greater below than above. Then only a minimal excess of pressure is required to empty the sausage skin completely.

Effective neutralization of gravitational distension of blood vessels is probably best effected, therefore, by immersion in water, which provides a graduated external counter-pressure which is greater below than above. It was, therefore, decided to attempt to neutralize the postural hypotension by immersing the patient in a warmed swimming pool. 
Effect of Immersion in Water Upon the Postural Hypotension Induced by Hexamethonium Bromide

Arrangements were made to have the water in the Physiotherapy Swimming Pool at $89^{\circ} \mathrm{F}$. and the room temperature $83^{\circ} \mathrm{F}$. Patients used to investigations were injected subcutaneously with sufficient H.M.B. to cause a substantial fall in the blood pressure. Preliminary readings were made in the horizontal and standing postures. The patients then advanced into the water and the relationship between the depth of immersion and the level of the blood pressure was determined. A typical result is shown in Fig. 3. Twelve such investigations were made. As the patient went deeper into the water the blood pressure rose to higher and higher levels until, when it was up to the chin, the blood pressure was only a little below that recorded before the administration of H.M.B. This return of the blood pressure almost to its original high level during immersion must be due to the counter-pressure of the water applied to the external surface of the body which neutralizes the gravitational increases of intravascular pressure that occurs in the legs on standing. When after H.M.B. injection the patient stands erect but out of the water, this gravitational increase causes distension of blood vessels (arterioles, capillaries and veins) in the lower part of the body and so a fall in blood pressure. This distension of blood vessels and the fall of blood pressure is prevented by immersion in water. Control tests made on the same patients without H.M.B. injections show little or no influence of water immersion upon the blood pressure level (Fig. 3).

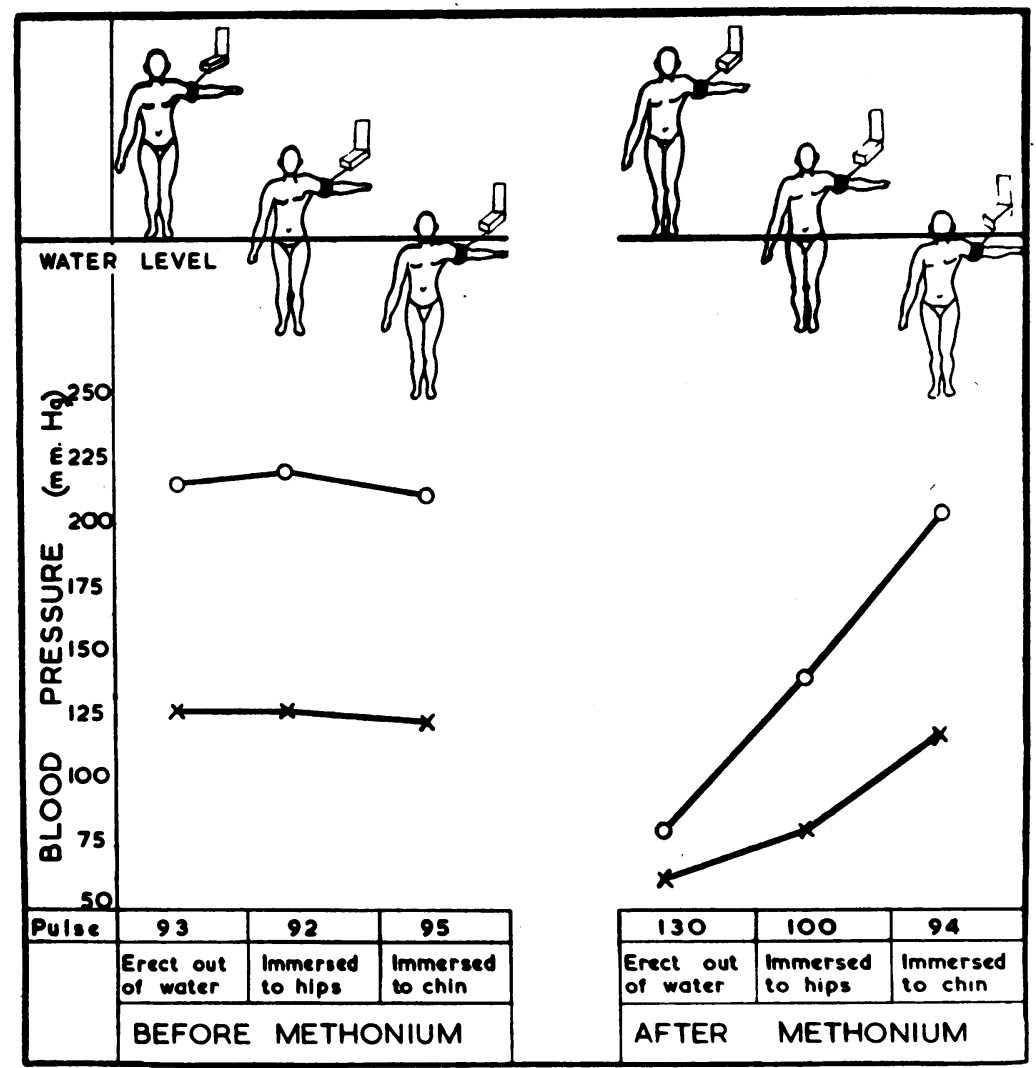

FIG. 3.-Effect upon the blood pressure of immersion of a patient to various depths in water, before and after injection of $H . M . B$.

After a suitable dose of P.M.B. or H.M.B. it is possible, by regulating the degree of immersion in warm water, to adjust the blood pressure of hypertensive patients at will to any desired level within a 
range that may be as wide as $130 \mathrm{~mm}$. $\mathrm{Hg}$ systolic and $50 \mathrm{~mm}$. diastolic. Such changes may be made in a few seconds, without symptoms, unless the pressure falls too low.

As was anticipated, the blood pressure after H.M.B. was not significantly affected by posture so long as complete immersion was maintained. Any fall in pressure obtained with large doses, despite immersion, was the same horizontal or erect.

\section{Evidence that Gravitational Effects Influence the Blood Pressure Levels in the Horizontal Posture after H.M.B.}

After injections of H.M.B. observations were made in which the patient was supported on a canvas stretcher and blood pressures were measured. Maintaining the horizontal posture, the patient was lowered into a warm bath so as to be immersed, leaving only the nose and mouth above water, and the blood pressure measured again. The arm in which it was measured was held level with the anterior aspect of the thorax. It was higher when the patient was immersed in the water than when out of the water; when the patient was half immersed, the blood pressure assumed intermediate levels. It would appear, therefore, that some gravitational distension of blood vessels takes place even in the horizontal posture.

Such observations raised the question of whether almost the entire blood-pressure lowering effect of H.M.B. might depend upon loss of the ability to resist this gravitational distension of blood vessels.

It was decided to study the effects of water immersion with larger doses of H.M.B. A large hospital bath was filled with water at $38^{\circ}$ or $39^{\circ} \mathrm{C}$. Intramuscular injections of H.M.B. were given until there was a good fall of blood pressure even in the horizontal posture. The patient was lowered into the bath on a canvas support, maintaining the horizontal posture and leaving the mouth, nose, and forehead out of the water. The arm used for blood pressure measurement was inclined so that the antecubital fossa and half of the cuff were out of the water. In all 6 observations it was observed that after large doses of H.M.B. the blood pressure, although raised by immersing the patient in water, was not restored to the pre-injection level, often remaining $30-50 \mathrm{~mm}$. in the systolic and $20 \mathrm{~mm}$. in the diastolic below the pre-injection level (Fig. 6). Blood pressure lowering by H.M.B. occurs even when gravitational increases in intravascular pressure of dependent blood vessels are counterbalanced by immersion in water. The water pressure will neutralize gravitational increases in intravascular pressure on both sides of the circulation.

\section{Effect of Water Immersion on the Postural Hypotension Following Surgical Sympathectomy}

As the postural hypotension induced by injections of H.M.B. is thought to be due to blocking of sympathetic ganglia, it seemed of interest to consider the effect of an external counter-pressure on the postural hypotension that occurs after successful sympathectomy for hypertension.

Protocol-Surgical Sympathectomy. Mr. G. S., aged 49, essential hypertension, pre-operative pressure 210/145. Following sympathectomy (D.10 to L.2 inclusive) his blood pressures out of the water were: lying 168/102, standing 120/104. Walking into the warm swimming pool his blood pressures were: at hip level 158/114, and at chin level 170/120. As the subject left the water the blood pressure fell.

In a patient with naturally occurring postural hypotension we confirmed the observation of Stead and Ebert (1941) that the postural fall of blood pressure can be prevented by immersion in water.

\section{Effect of Applying a Uniform Positive Pressure to the Body Surface Below Nipple Level}

When the body below the nipple level is enclosed in an air pressure chamber it is possible to study the effect upon the hypotensive action of H.M.B. of applying a uniform external counterpressure of $20-60 \mathrm{~mm}$. $\mathrm{Hg}$ to those parts of the body that lie within the pressure chamber. Investigations were made with a patient in the upright posture so that on injection with H.M.B. the blood pressure fell to a low level. It is clear that after H.M.B. the application of an external counter-pressure with compressed air may raise the blood pressure significantly, and may restore a patient who is feeling faint (Fig. 4). 


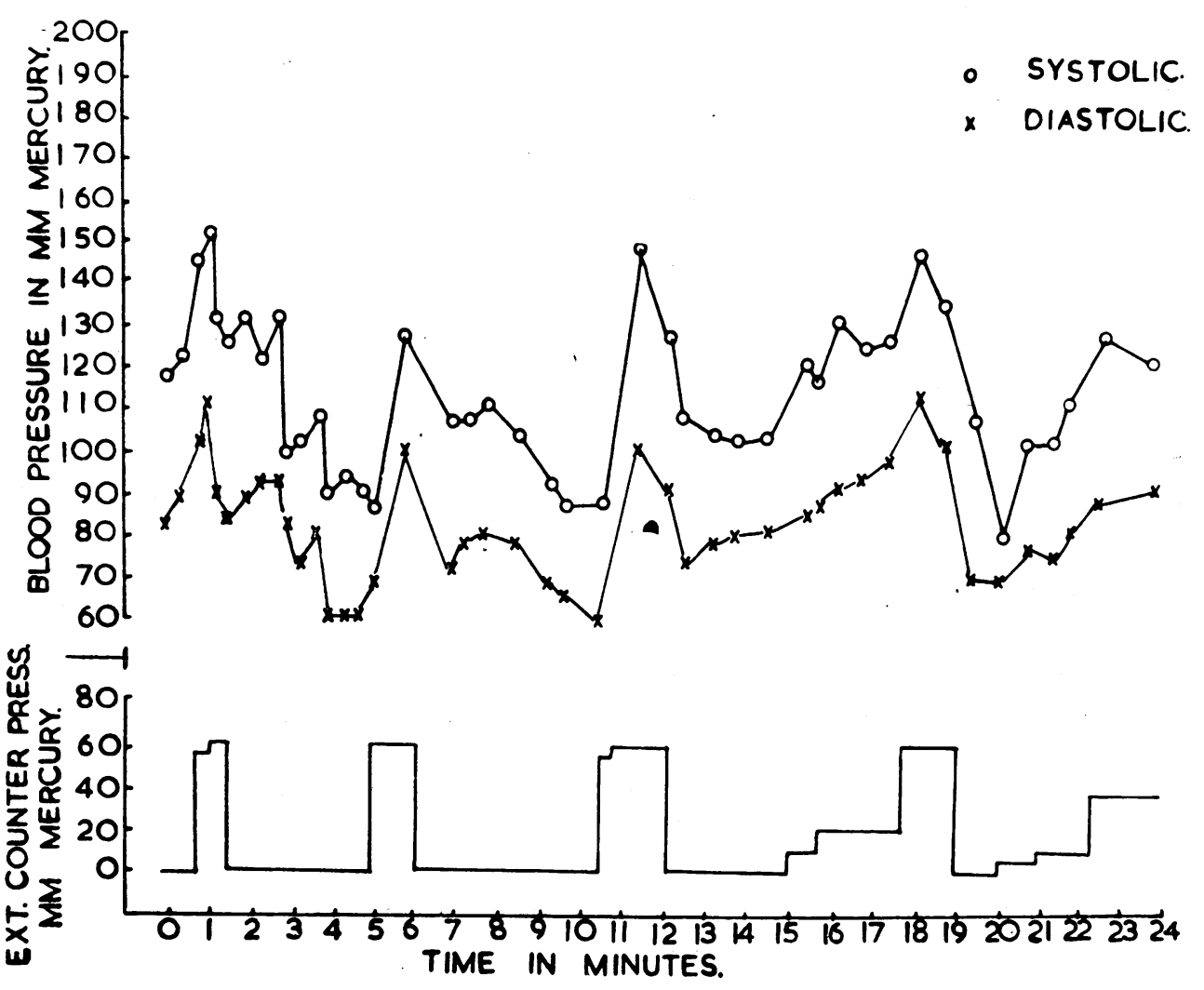

Fig. 4.-Effect of applying a positive air pressure to the body surface below the nipple level after an injection of $H . M . B$.

\section{Effect of Applying a Uniform Negative Pressure to the Body Surface Below Nipple Level}

When the body below nipple level is enclosed in a pressure chamber, the application of a negative pressure may reduce the blood pressure even when no H.M.B. has been administered (Fig. 5). The return of the pressure in the chamber to the atmospheric level is associated with restoration of the original blood pressure. After administration of H.M.B. the effect of a negative pressure applied to the lower two-thirds of the patient's body is greatly enhanced and, with sufficient negative pressure, a patient may be brought near to fainting within a few seconds. During the application of a negative pressure, either without or with the previous administration of H.M.B., a distinct fall in the venous pressure was observed in the neck veins. The fall of arterial pressure is greater after H.M.B., probably because arterioles, capillaries, and veins are less able to resist an expanding force.

The effect of applying a negative pressure to the entire body is not the same. A hypertensive patient and an observer were enclosed in an Air Force decompression chamber and the pressure within the chamber reduced to $236 \mathrm{~mm}$. $\mathrm{Hg}$ below atmospheric, corresponding to a height of $10,000 \mathrm{ft}$. As expected, there was no appreciable effect upon the blood pressure level.

\section{Effect of Immersion in Water on the Blood Pressure Lowering Effect of Nitroglycerine}

It is generally thought that nitroglycerine acts by direct depression of the smooth muscle of blood vessels. It has been pointed out by Weiss et al. (1937), that a degree of postural hypotension occurs during the action of nitroglycerine. It was, therefore, of interest to decide whether the fall of blood pressure from $1 / 200$ or $1 / 100$ th grain of trinitrin, dissolved under the tongue, with the 


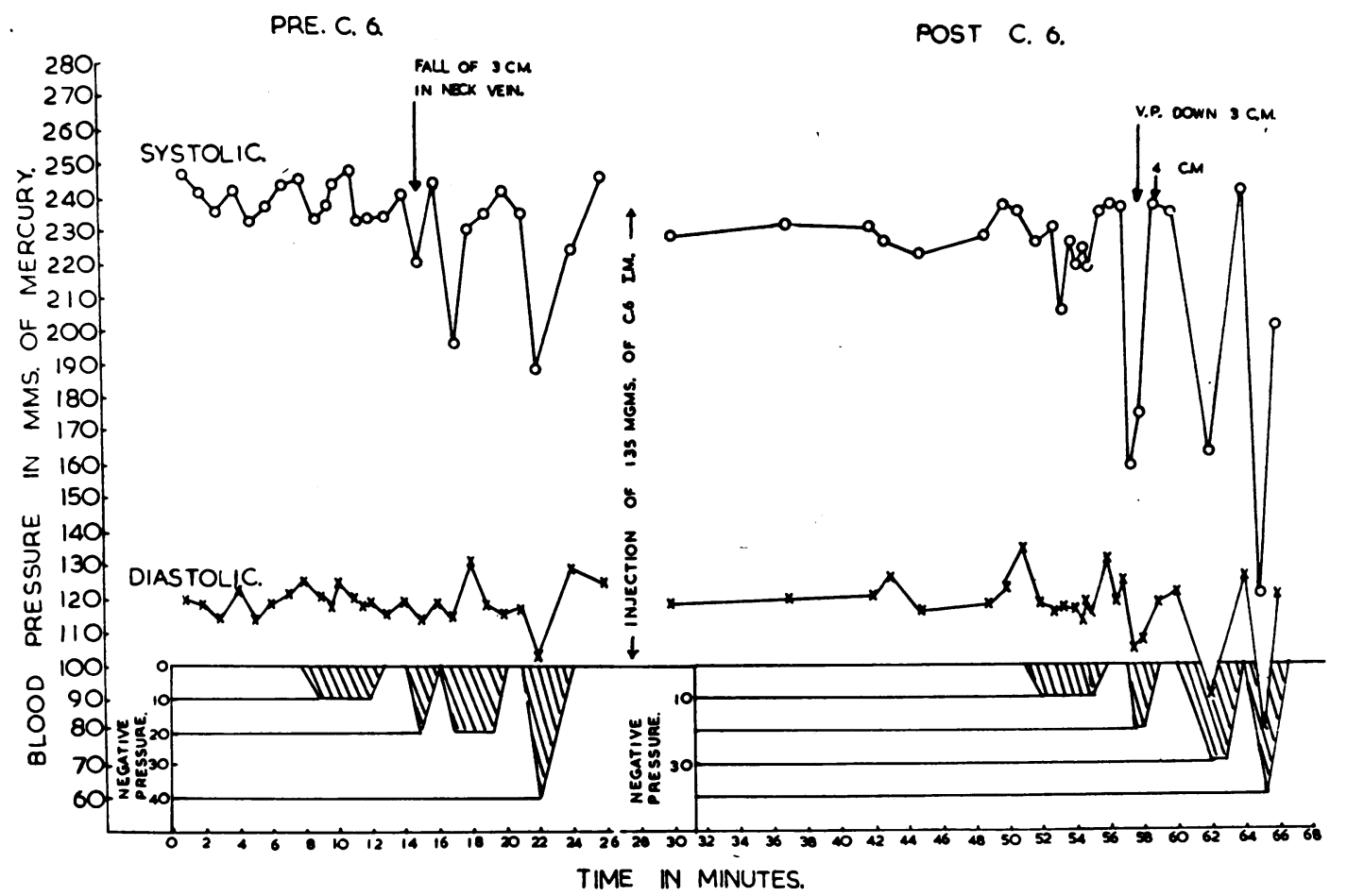

FIG. 5.-Effect of applying a negative air pressure to the body surface below the nipple level, before and after giving H.M.B. The degrees of negative air pressure are in $\mathrm{mm}$. of mercury.

subject in the erect posture, could be counteracted by immersion in water. Four experiments were performed, of which one is illustrated in Fig. 7.

With the doses employed, the blood pressure scarcely fell when the subject was immersed up to the shoulders in a warm swimming bath, whereas standing by the side of the swimming bath the same dose dropped the blood pressure to a low level and in some cases the subject felt faint and had to be laid flat.

\section{Some Effects of H.M.B. on the Blood Flow}

It has already been stated by Kay and Smith (1950) that, even with the blood pressure reduced after H.M.B., the skin temperature in the arms, and especially in the legs, may rise. This suggests some dilatation of the arterioles in the part showing a temperature increase.

In hypertensive patients it was found that forehead temperature tended to remain unaltered or fall, and arm and leg temperatures to remain unaltered or more usually to rise. As the blood pressure falls after H.M.B. it follows that, if the limb temperature does not fall with it, then the peripheral resistance in the limb must have been decreased.

Cardiac Output in Relation to Blood Pressure Lowering by H.M.B.

Some preliminary observations (Restall and Smirk, 1950) suggested, on the basis of ballistocardiograms, that during blood pressure reduction by $H . M . B$. the heart's output at rest might be either increased or diminished. It seemed of interest to consider whether the blood pressure fall would be maintained if the heart's output were increased. Ballistocardiograms were taken on a hypertensive patient while at rest in the sitting posture first before and then after performing an exercise suited to his physical capacity. Ordinarily exercise was the ascent of some 20 to 40 steps. The patient then received a suitable injection of H.M.B. and the observation was repeated. The 


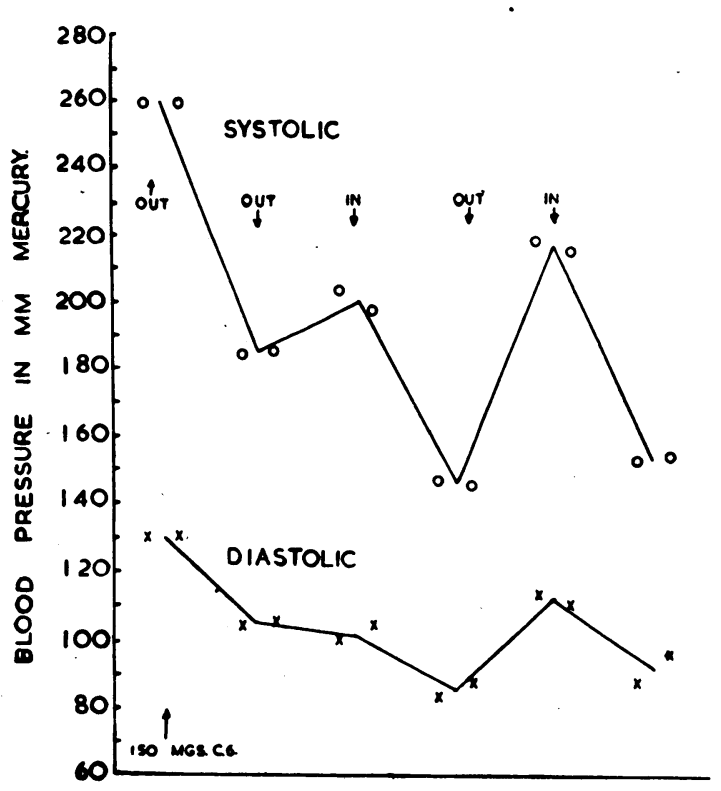

FIG. 6.-Effect of immersion in warm water on the blood pressure of a patient who is maintained in the horizontal posture throughout the test. The patient has received a subcutaneous injection of $150 \mathrm{mg}$. of H.M.B. (In and out refer to the presence or absence of water immersion at the time of measuring the blood pressure and an additional "out" should have been inserted above the last circle.)

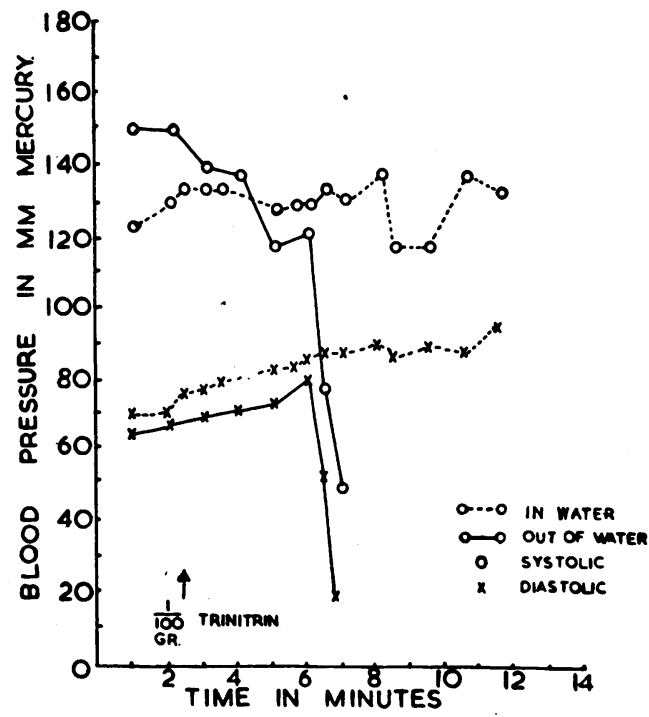

FIG. 7.-Effect of immersing the subject in water upon the hypotensive action of trinitrin.

blood pressure was measured at various stages in the course of the test. Four such experiments were performed.

Although ballistocardiograms may be open to suspicion as providing an absolute measurement of the cardiac output, there can be little doubt that gross changes in the cardiac output in the same subject can be reported on with some confidence. It will be seen that the increase of the cardiac output after exercise is not prevented by H.M.B. (Fig. 8), for both the rate of the heart and the amplitude of the deflections may be augmented simultaneously. In the resting state immediately following exercise, and at a time when the cardiac output is increased, the blood pressure after H.M.B. remains at a low level. On the basis of such results it is hoped in the discussion to comment upon the relationship between cardiac output and peripheral resistance.

\section{Discussion}

When P.M.B. or H.M.B. are given in sufficient amounts they reduce the blood pressure even when the patient is in the horizontal posture. Their most prominent circulatory effect, however, is to induce a postural hypotension and as the subject approximates to the vertical the blood pressure falls further. The systolic fall is greater, the pulse usually accelerates and, with the patient sitting, the leg volume increases. Venous pressures fall, both in health and in congestive heart failure patients, and in 4 out of 5 of the latter a decrease in breathlessness occurred simultaneously.

An attempt to limit the postural blood pressure fall by elastic binding of the legs and abdomen led us to study the effect of a measurable hydrostatic counter-pressure obtained by immersing the patient in a warm swimming bath. The effects were dramatic, for as the patient walked on into the deeper part of the swimming pool so the blood pressure rose until, in some cases, it was only a little below the premedication hypertensive level. Rises of blood pressure of 130 systolic and 70 diastolic have been induced in this way, and by regulating the depth of immersion almost any 


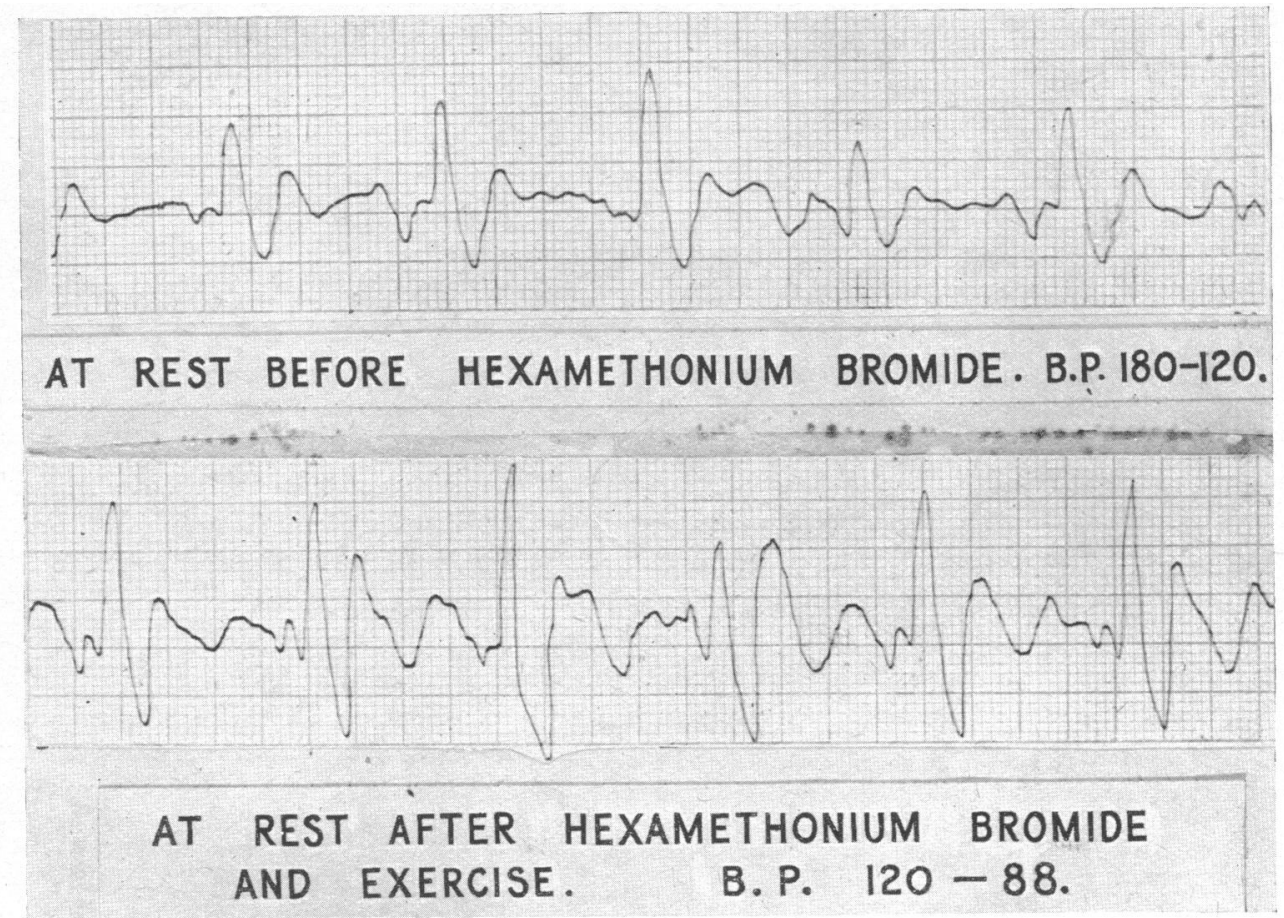

FIG. 8.-Ballistocardiographic evidence that blood pressure lowering from H.M.B. persists in the presence of a raised cardiac output. The blood pressure at rest was $180 / 120$ before H.M.B., and during rest after exercise was $120 / 88$ after H.M.B. The corresponding ballistocardiograms are shown.

selected blood pressure within the range (either systolic or diastolic) can be obtained. If, before immersion, the pulse had accelerated as the blood pressure fell, then, on immersion, the pulse usually returned to its original rate as the blood pressure rose. Pulse rate is more closely related to blood pressure than to the amount of drug injected. In the swimming bath, as one might expect, there is practically no difference between a patient's pressure in the horizontal and in the vertical positions, provided that in both positions the patient is under water except for the parts above the level of the chin.

These investigations prompted others in which the patient stood in an air pressure chamber with an airtight joint at nipple level. It was found that a positive pressure applied to the body below the level of the nipples would partly counteract the hypotension that develops in the vertical posture after H.M.B. If, however, the device be used to impose a negative pressure upon the body below the nipple level then in normal and hypertensive people, even in the horizontal posture and without medication, considerable blood pressure reductions occur when the external pressure is reduced. A fall in venous pressure occurs concurrently. After administration of H.M.B. the effects are enhanced, bigger falls being obtained with lesser negative pressures. Negative pressure applied in this way is to some extent analogous to the effects of " $G$," as when an aircraft makes a sharp turn.

The great influence of posture on the blood pressure following H.M.B., the nearly complete reversal of this effect by the external graduated counter-pressure of water, and the potentiation of the effect by a negative external pressure deserve an explanation. In our water immersion experiment the internal hydrostatic pressure on the venous side corresponds closely to the external counter-pressure of the surrounding water when immersion is to manubrial level (Smirk, 1936; Walker and Longland, 1950). We thought at first that the procedures described altered blood pressure by their effects on the amount of blood pooled in dependent parts, but later regarded their 
effects on the calibre of arterioles, and hence on the peripheral resistance, as of greater importance. In perfusing isolated rat limbs we have noted that if the rate of perfusion is doubled the perfusion pressure is not doubled (cf. Poiseuille's Law). The reason is that the arterioles are stretched by the increased internal pressure leading to a decrease in peripheral resistance.

When a healthy man stands up there is a brief fall of blood pressure, probably due to distension of blood vessels following the gravitational increase of pressure within them. This quickly resumes its original level by reflex action through the carotid sinus and elsewhere. After H.M.B. the body loses some of its capacity to counteract this gravity-pressure dilatation of vessels because of the sympathetic paths having been made less effective. A similar loss occurs after nitroglycerine due to its direct inhibitory effect making the plain muscle less able to resist the rise of intravascular pressure. So-called "postural hypotension" would be better termed "gravitational hypotension," since it can be shown to be present to some degree in the horizontal as well as in the vertical posture.

Gravitational effects, though important, are not the whole explanation of the fall of blood pressure because sufficiently large doses of H.M.B. still cause distinctive blood pressure falls when gravity effects are counteracted by immersion in a warm bath. The effect of H.M.B., decreasing the ability of vessel walls to withstand high intravascular pressures, probably explains why greater pressure falls occur when the initial pressure is high than when it is normal.

Confirming Kay and Smith (1950), we found that although the blood pressure falls after H.M.B. injections, the limb temperatures rise or are unaltered; hence the peripheral resistance in the limbs must be decreased. Evidently there is local arteriolar dilatation. Such local increase of blood flow may occur at the expense of other vascular beds, but a general increase requires increased cardiac output.

Under the varying conditions of everyday life ambulant patients, whether on H.M.B. treatment or not, exhibit changes in both cardiac output and peripheral resistance, and maintenance of a fall of blood pressure demands that the change in the relationship between them should be preserved under various physiological conditions. Were the blood pressure fall under H.M.B. dependent upon a simple change such as a decrease in the cardiac output, then the blood pressure should return to a high level if the cardiac output were raised by exercise. It has been shown that in a hypertensive under H.M.B. the cardiac output may be increased above the resting output by exercise without loss of the hypotensive action.

\section{CONCLUSIONS}

Hexamethonium bromide (H.M.B.) alters the relationship between cardiac output and peripheral resistance in such a way that, for any given cardiac output, the peripheral resistance is less than it would be in the absence of H.M.B. This altered relationship, though it may be modified, is not abolished by mild exercise, meals, and emotion.

The gravitational (so-called " postural ") hypotension induced by H.M.B. is demonstrable in the horizontal as well as the vertical posture and is due to decrease in the capacity of vessel walls of dependent parts to contract and thereby resist gravity-pressure dilatation. This impaired homeostasis arises from less effective action of sympathetic paths.

We acknowledge with gratitude the skilful technical assistance of Miss J. Rivers. We are much indebted to Miss M. Walsh and Miss M. Poppelwell for secretarial assistance, to Mr. E. R. Macdonald for photographs, and to the physicians of the Dunedin Hospital and many practitioners who have referred patients to us.

The expenses of the investigation were defrayed by the Medical Research Council of New Zealand and the Otago University.

\section{REFERENCES}

Arnold, P., Goetz, R. H., and Rosenheim, M. L. (1949). Lancet, 2, 408.

and Rosenheim, M. L. (1949). Lancet, $2,321$.

Balaban, I. E., Levy, M. B., and Wilde, B. E. (1949). J. Pharmacy, Lond., 1, 603.

Barlow, R. B., and Ing, H. R. (1948a). Nature, Lond., 161, 718.

(1948b). Brit. J. Pharmacol., 3, 298. 
Burn, J. H., and Dale, H. H. (1915). J. Pharm. Exper. Therap., 6, 417.

Burt, C. C., and Graham, A. J. P. (1950). Brit. med. J., 1, 455.

Campbell, A., and Robertson, E. (1950). Brit. med. J., 2, 804.

Cohen, L. (1936). J. Lab. clin. Med., 22, 94.

Crum-Brown, A., and Fraser, T. R. (1868). J. Anat. Physiol. 1 (second series), 224.

Drury, A. N., and Jones, N. W. (1927). Heart, 14, 55.

Kay, A. W., and Smith, A. N. (1950). Brit. med. J., 1, 460.

Lewis, T. (1943). Diseases of the Heart, p. 10. Macmillan, London.

Organe, G., Paton, W. D. M., and Zaimis, E. J. (1949). Lancet, 1, 21.

Paton, W. D. M., and Zaimis, E. J. (1948a). Nature, Lond., 161, 718. (1948b). Nature, Lond., 162, 810.

- (1949). Brit. J. Pharmacol., 4, 381.

Restall, P. A., and Smirk, F. H. (1950). N.Z. med. J., 49, 206.

Saville, S. (1950). Lancet, 2, 358.

Smirk, F. H. (1936). Clin. Sci., 2, 317. (1950a). Lancet, 2, 477. (1950b). N.Z. med. J., 49, 637.

and Alstad, K. S. (1951). Brit. med. J., 1, 1217.

Stead, E. A., and Ebert, R. V. (1941). Arch. intern. Med., 67, 546.

Turner, R. (1950). Lancet, 2, 353.

Walker, A. J., and Longland, C. J. (1950). Clin. Sci., 9, 101.

Weiss, S., Wilkins, R. W., and Haynes, F. W. (1937). J. Clin. Invest., 16, 73. 